\title{
The Effect of Phosphate Mixing on Structural, Spectroscopic, Mechanical and Optical Properties of Zinc tris Thiourea Sulphate (ZTS) Single Crystals
}

\author{
A. Puhal Raj and C. Ramachandra Raja* \\ Government Arts College (Autonomous), Kumbakonam-612001, Tamilnadu, India. \\ *Corresponding Author: crraja_phy@yahoo.com.
}

\begin{abstract}
Zinc tris Thiourea Sulphate (ZTS) is a semi organic non-linear optical crystal. The ZTS and phosphate mixed (in different mol \%) ZTS crystals have been grown from aqueous solution using the slow evaporation method. Single crystal XRD were carried out for ZTS and phosphate mixed ZTS crystals to determine cell parameters. FTIR analysis identified modes of vibrations of different molecular groups and confirms the presence of phosphate ion. The UV-Vis-NIR spectrum shows that the material has wide optical transparency in the entire visible region. The second harmonic generation (SHG) was confirmed by Kurtz powder method. It is found that 10 mol \% of phosphate mixed ZTS crystal has better SHG efficiency than pure ZTS crystal. The Vicker microhardness test was carried out on the grown crystals and Vicker's Hardness Number was found increase with increase in phosphate mixing.
\end{abstract}

Keywords: Phosphate mixed zinc tris thiourea sulphate; Second harmonic generation; NLO material.

\section{INTRODUCTION}

A non linear optical (NLO) material plays a crucial role in the laser technology, optical communication and electro optic modulation [1, 2]. Materials with good non linear optical susceptibilities and high laser damage threshold value are needed for these applications. There are many works carried out to synthesis NLO materials. In the present work, improving quality of a NLO material Zinc tris Thiourea Sulphate (ZTS) crystal with molecular formula $\mathrm{Zn}\left(\mathrm{NH}_{2} \mathrm{CSNH}_{2}\right)_{3} \mathrm{SO}_{4}$ has been done successfully by mixing of phosphate. ZTS belongs to orthorhombic system with point group $\mathrm{mm} 2$ and space group Pca2 ${ }_{1}$. SHG efficiency of ZTS is 
1.2 times is that of KDP [3]. The growth and characterization of ZTS were reported in a number of recent papers [4-6]. Jayavel et al reported substitution of phosphate with ZTS improves its properties [7]. It is of interest to us to investigate the effect of high level mixing of phosphate ion with ZTS. Hence, we report the property change of ZTS crystals due to high level phosphate mixing.

\section{EXPERIMENTAL}

\subsection{Synthesis}

The ZTS was synthesized from zinc sulphate heptahydrate and thiourea taken in the ratio of 1:3 by the following reaction

$$
\mathrm{ZnSO}_{4} .7 \mathrm{H}_{2} \mathrm{O}+3 \mathrm{NH}_{2} \mathrm{CSNH}_{2} \rightarrow \mathrm{Zn}\left(\mathrm{NH}_{2} \mathrm{CSNH}_{2}\right)_{3} \mathrm{SO}_{4}+7 \mathrm{H}_{2} \mathrm{O}
$$

The solution of zinc sulphate heptahydrate was added to the solution of thiourea, the mixture had to be stirred vigorously to avoid precipitation of other phases. The resultant precipitate of ZTS was dried. The salt was purified by repeated recrystallization process by using double distilled water as solvent. The ZTS solution was prepared and maintained at $36^{\circ} \mathrm{C}$ in a constant temperature bath with an accuracy of $\pm 0.01^{\circ} \mathrm{C}$ with continuous stirring to ensure homogeneous temperature concentration over the entire volume of the solution. The transparent colourless ZTS single crystals were grown in a period of 40 days by using slow evaporation technique. In the ZTS solution, the phosphoric acid was dissolved with different molar concentration (10 and 25 mol \%). Phosphate mixed ZTS solutions are also allowed to slow evaporation by using the above condition. The good quality phosphate mixed ZTS crystals were harvested in the same period.

\subsection{Characterization}

The grown ZTS and 10 and 25 mol \% of phosphate mixed ZTS single crystals were subjected to various characterization techniques like single crystal XRD, Fourier transform infrared (FTIR), UV-Vis-NIR, microhardness and non linear optical studies. The single crystals of ZTS and 10 and $25 \mathrm{~mol} \%$ of phosphate mixed ZTS have been subjected to X-ray diffraction studies using an ENRAF NONIUS CAD4 X-ray diffractometer to determine the unit cell parameters. The FTIR spectra were carried out using Perkin Elmer RX1 spectrometer. The UV-Vis-NIR spectral studies were studied using Lambda 35 spectrometer in the range 190-1100 nm. The Vickers hardness measurement was made on the crystals using Shimadzu (Japan) HMV-2 hardness tester. SHG test has been studied by Kurtz powder technique.

\section{RESULTS AND DISCUSSION}

\subsection{Single Crystal X-ray Diffraction}


Table 1: Unit cell parameters of pure ZTS and phosphate mixed ZTS single crystals.

\begin{tabular}{ccccc}
\hline Crystal & $\mathrm{a}(\AA)$ & $\mathrm{b}(\AA)$ & $\mathrm{c}(\AA)$ & $\mathrm{V}\left(\AA^{3}\right)$ \\
\hline Pure ZTS & 11.141 & 7.769 & 15.501 & 1342 \\
$10 \mathrm{~mol} \%$ of $\mathrm{PO}_{4}+$ ZTS & 11.141 & 7.770 & 15.537 & 1345 \\
$25 \mathrm{~mol} \%$ of $\mathrm{PO}_{4}+$ ZTS & 11.156 & 7.786 & 15.526 & 1348 \\
\hline
\end{tabular}

The cell parameters of pure ZTS and phosphate mixed ZTS crystals are given in table 1 . The values of $\alpha, \beta$ and $\gamma$ are same for pure and phosphate mixed ZTS crystals which is indicating no change in the orthorhombic system due to mixing of phosphate ions. Cell volume of pure ZTS and 10 and $25 \mathrm{~mol} \%$ of phosphate mixed ZTS are $1342 \AA^{3}, 1345 \AA^{3}$ and $1348 \AA^{3}$ respectively. There is a slight increase in the cell volume of phosphate mixed ZTS when compared to pure ZTS crystals. This shows that phosphate ions are entered into the ZTS crystal lattice.

\subsection{FTIR Spectral Analysis}

In ZTS compound, there are three thiourea groups and one sulphate ion. Each thiourea group consists of one carbon atom bonding to one sulphur and two nitrogen atoms. Each of the nitrogen atoms in thiourea is connected to two hydrogen atoms. Zinc ion is tetrahedraly coordinated to three sulphur atoms of thiourea and to an oxygen atom of sulphate ion [8]. The FTIR spectra of pure ZTS and 10 and $25 \mathrm{~mol} \%$ of phosphate mixed ZTS were recorded in the range 450-4000 $\mathrm{cm}^{-1}$ and they are shown in Fig. 1(a-c). The spectra of ZTS and phosphate mixed ZTS crystals can be interpreted as follows: The N-H absorption frequency in the region between $3000 \mathrm{~cm}^{-}$ ${ }^{1}$ and $4000 \mathrm{~cm}^{-1}$ arises due to symmetric and asymmetric vibration of $\mathrm{NH}_{2}$ group of the zinc coordinated thiourea [8]. The bending vibration of $\mathrm{NH}_{2}$ was observed around $1627 \mathrm{~cm}^{-1}$ [9]. The $\mathrm{C}-\mathrm{N}$ and N-C-N stretching vibration are observed near $1114 \mathrm{~cm}^{-1}$ and $1510 \mathrm{~cm}^{-1}$, respectively [8].

The symmetric and asymmetric stretching vibration of $\mathrm{C}=\mathrm{S}$ was observed around $714 \mathrm{~cm}^{-1}$ and $1401 \mathrm{~cm}^{-1}$, respectively [10]. The symmetric and asymmetric bending vibration of N-C-S was observed near $474 \mathrm{~cm}^{-1}$ and $617 \mathrm{~cm}^{-1}$ respectively. The asymmetric bending vibration of N-C-N was observed near $530 \mathrm{~cm}^{-1}$ [8]. The presence of peaks near $1000 \mathrm{~cm}^{-1}$ confirms the presence of sulphate ion in the coordination sphere. The observed additional peaks near $1200 \mathrm{~cm}^{-1}$ clearly confirms the presence of phosphate in the coordination sphere [7]. The observed vibrational spectra of phosphate mixed ZTS crystals are similar to the vibrational spectra of pure ZTS crystal and only very slight frequency shifts are observed. The tentative assignments of recorded FTIR spectra are given in table 2 . 

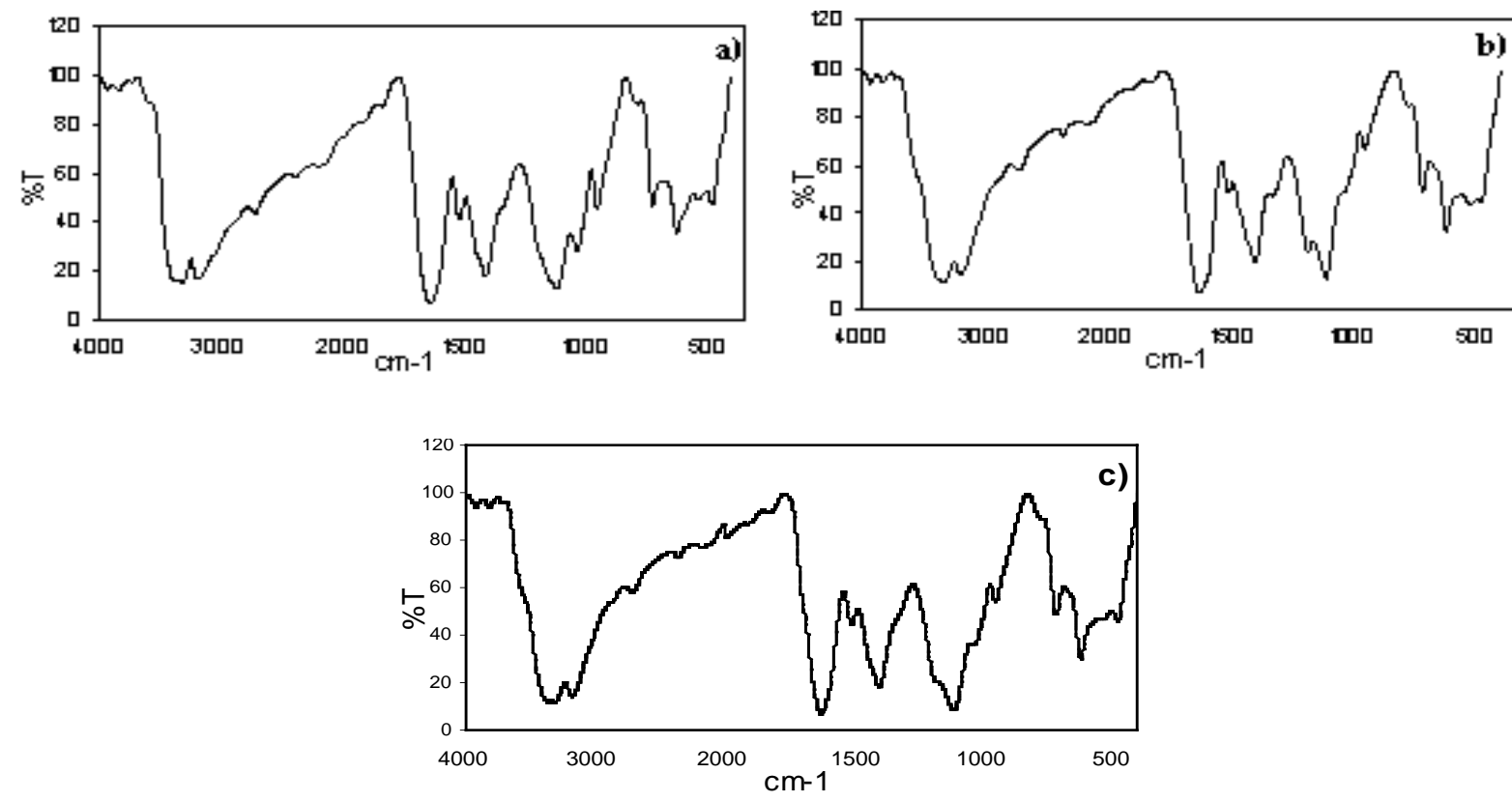

Fig. 1: FTIR spectra for a) pure ZTS and b) 10, c) 25 mol \% of phosphate mixed ZTS.

Table 2: Comparison of vibrational modes of pure ZTS and phosphate mixed ZTS. (v-bond stretching; s-symmetric; as-asymmetric; $\delta$-deformation).

\begin{tabular}{|c|c|c|c|}
\hline Pure ZTS $\left(\mathrm{cm}^{-1}\right)$ & $\begin{array}{l}10 \mathrm{~mol} \% \mathrm{PO}_{4}+ \\
\mathrm{ZTS}\left(\mathrm{cm}^{-1}\right)\end{array}$ & $\begin{array}{l}25 \mathrm{~mol} \mathrm{\%} \mathrm{PO}_{4}+ \\
\mathrm{ZTS}\left(\mathrm{cm}^{-1}\right)\end{array}$ & Assignments \\
\hline 474 & 479 & 475 & $\delta \mathrm{s}(\mathrm{N}-\mathrm{C}-\mathrm{S})$ \\
\hline 530 & 524 & 530 & $\delta \operatorname{as}(\mathrm{N}-\mathrm{C}-\mathrm{N})$ \\
\hline 617 & 619 & 617 & $\delta$ as (N-C-S) \\
\hline 714 & 715 & 714 & $v s(\mathrm{C}=\mathrm{S})$ \\
\hline 1114 & 1109 & 1111 & $v(\mathrm{C}-\mathrm{N})$ \\
\hline 1401 & 1400 & 1400 & $\operatorname{vas}(\mathrm{C}=\mathrm{S})$ \\
\hline 1510 & 1507 & 1507 & $v(\mathrm{~N}-\mathrm{C}-\mathrm{N})$ \\
\hline 1627 & 1626 & 1625 & $\delta\left(\mathrm{NH}_{2}\right)$ \\
\hline 3179 & 3184 & 3186 & $v \mathrm{~s}\left(\mathrm{NH}_{2}\right)$ \\
\hline 3310 & 3318 & 3311 & $v \mathrm{~s}\left(\mathrm{NH}_{2}\right)$ \\
\hline 3825 & 3823 & 3822 & $\operatorname{vas}\left(\mathrm{NH}_{2}\right)$ \\
\hline 3924 & 3923 & 3928 & $\operatorname{vas}\left(\mathrm{NH}_{2}\right)$ \\
\hline
\end{tabular}

\subsection{UV-Vis-Nir Spectral Analysis}

The optical absorption spectra of ZTS and phosphate mixed ZTS single crystals were recorded in the range 190-1100 $\mathrm{nm}$. These are shown in the Fig. 2(a-c). The wide transmission in the region 
$300-1100 \mathrm{~nm}$ is an advantage as it is the key requirement for NLO application. The spectra of absorption show that all of three crystals have lower cut off wavelength at around $300 \mathrm{~nm}$. The peak in the range 195 to $300 \mathrm{~nm}$, in pure ZTS and phosphate mixed ZTS crystals is due to $\pi$ - $\pi^{*}$ transitions of thiourea.
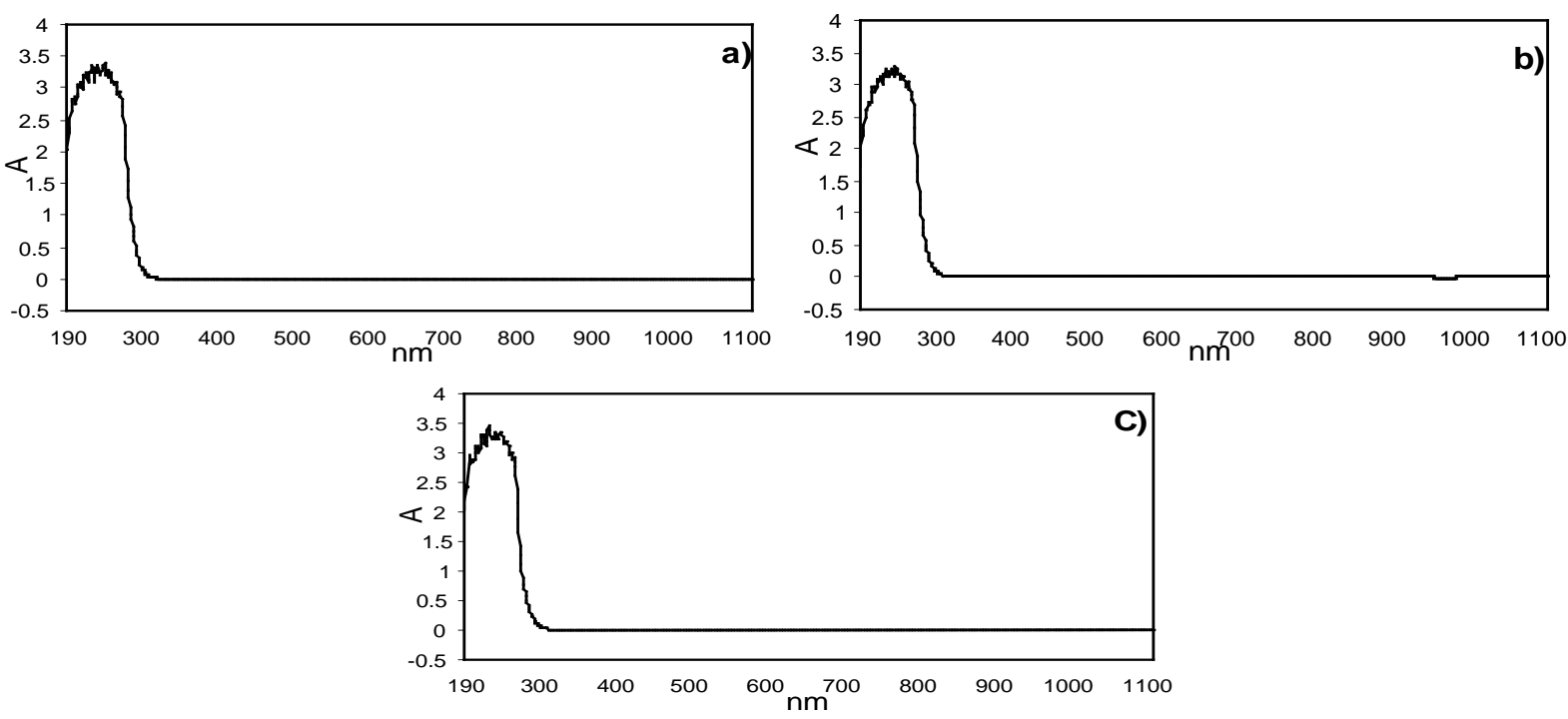

Fig. 2: UV absorbance spectra for a) pure ZTS and b) 10, c) 25 mol \% of phosphate mixed ZTS.

\subsection{Microhardness Studies}

The Vicker microhardness test was performed on ZTS and phosphate mixed ZTS single crystals. Hardness measurements were taken for different applied loads. Vicker's Hardness Number (VHN) was calculated for the samples by using the relation $\mathrm{Hv}=1.8544 \mathrm{P} / \mathrm{d}^{2} \mathrm{Kg} \mathrm{mm}{ }^{-2}$. Here, $\mathrm{Hv}$ is Vicker's Hardness Number, $\mathrm{P}$ is applied load in $\mathrm{Kg}$ and $\mathrm{d}$ is average diagonal length in $\mathrm{mm}$. The tests showed that phosphates mixed ZTS crystals are stronger than pure ZTS crystals. Fig. 3. shows value of $\mathrm{Hv}$ increases with increase on load up to $200 \mathrm{gm}$ and then attains saturation.

Increases in hardness value of phosphate mixed ZTS crystals are due to the addition of phosphate in the crystal lattice. The degree of negative charge of phosphate ion is high and it improves bonding between phosphate ion and others. Hardness values are increased when the percentage of phosphate addition increases. The increase in the hardness value of phosphate mixed ZTS single crystals proves it to be a good engineering material for the fabrication of devices for NLO process. 


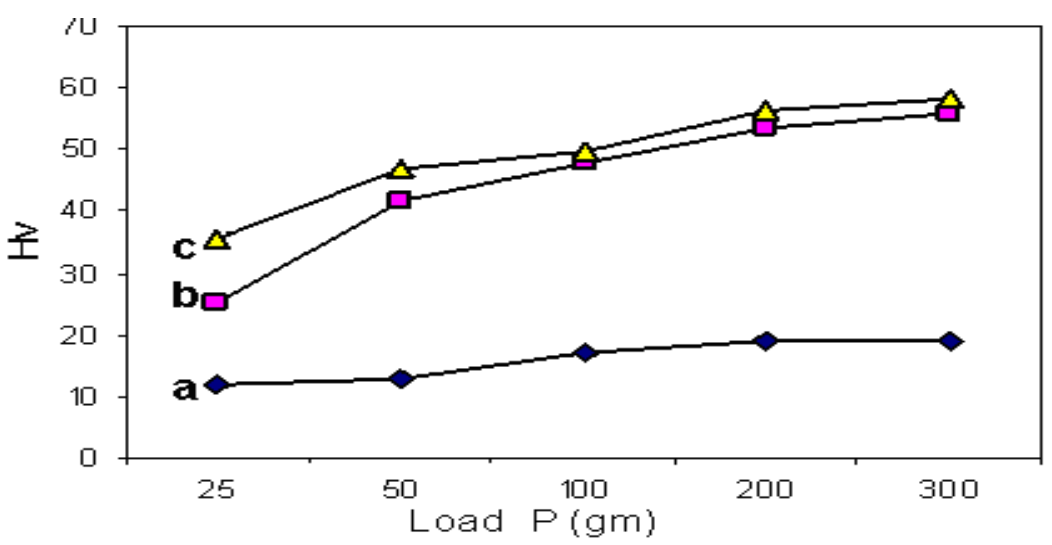

Fig. 3: Variation of microhardness for a) pure ZTS and b) 10, c) $25 \mathrm{~mol} \%$ of phosphate mixed ZTS.

\subsection{Second Harmonic Generation Studies}

Second Harmonic Generation (SHG) test for the powder samples of pure ZTS and phosphate mixed ZTS have been studied by kurtz powder technique. In this method the powder samples were illuminated using Nd:YAG laser emitting a fundamental wavelength of $1064 \mathrm{~nm}$. The second harmonic generation was confirmed by the emission of green radiation by the crystal samples with the output power of 27, 32 and $25 \mathrm{mV}$ for pure ZTS and 10 and $25 \mathrm{~mol} \%$ of phosphate mixed ZTS respectively. The results are shown in table 3 . The $\pi$ orbital electron delocalization in thiourea due to mesomeric effect is the reason for the non linear optical response of ZTS and phosphate mixed ZTS crystals. As the degree of negative charge on oxygen of phosphate is more than oxygen of sulphate, phosphate may coordinate better than sulphate. This shows that the bonding between the zinc and oxygen of phosphate is stronger. This may weaken the interaction of zinc with thiourea in phosphate mixed ZTS and hence the delocalization of the electronic cloud between the zinc and sulphur of thourea is hindered. This could be the reason for the change in the SHG efficiency of phosphate mixed ZTS crystals compared to pure ZTS crystal. Efficiency of NLO property increases upto $18 \%$ in 10 mol \% of phosphate mixed ZTS crystal compared to pure ZTS.

Table 3: SHG efficiency of pure ZTS and $\mathrm{PO}_{4}$ mixed ZTS single crystals.

\begin{tabular}{cl}
\hline Crystal & SHG efficiency \\
\hline Pure ZTS & $27 \mathrm{mV}$ \\
$10 \mathrm{~mol} \mathrm{\%} \mathrm{of} \mathrm{PO}_{4}+$ ZTS & $32 \mathrm{mV}$ \\
$25 \mathrm{~mol} \mathrm{\%} \mathrm{of} \mathrm{PO}_{4}+$ ZTS & $25 \mathrm{mV}$ \\
\hline
\end{tabular}




\section{CONCLUSION}

Single crystals of pure ZTS and 10 and $25 \mathrm{~mol} \%$ of phosphate mixed ZTS have been grown by slow evaporation method. Single crystal XRD studies reveal that no structural change in phosphate mixed ZTS crystals. The presence of functional groups in grown crystals is identified by FTIR spectral analysis. UV-Vis-NIR spectra shows broad transparency of pure ZTS and phosphate mixed ZTS crystals lies between $300 \mathrm{~nm}$ and $1100 \mathrm{~nm}$. Vicker hardness test show increase in the hardness value of phosphate mixed ZTS crystals due to addition of phosphate in ZTS lattice. SHG test reveals that $10 \mathrm{~mol} \%$ of phosphate mixed ZTS crystal has greater SHG efficiency than pure ZTS. Hence, it is concluded that due to its wide transparency range, high hardness and greater SHG conversion efficiency $10 \mathrm{~mol} \%$ of phosphate mixed ZTS crystal can be a good engineering material for fabrication of nonlinear optical devices.

\section{ACKNOWLEDGEMENTS}

The authors are very grateful to Dr. P.K. Das, Indian Institute of Science, Bangalore (India) for measurement of SHG efficiency and IIT, Chennai for FTIR spectrum. The authors thank Madurai Kamaraj University, Madurai for single crystal XRD and St. Joseph’s College, Tiruchirappalli for microhardness studies.

\section{REFERENCES}

1. Marcy, H. O., Warren, L. F., Webb, M. S., Ebbers, C. A., Velsko, S. P., Kennady, G. C., and Catella G. C., 1992, "Second-harmonic generation in zinc tris(thiourea) sulphate" Applied Opts., Vol. 31, Ed. 24, pp. 5051-5060.

2. Wang X. Q., Xu D., Yuan D. R., Tian Y. P., Yu W. T., Sun S. Y., Yang Z. H., fang Q., Lu M. K., Yan Y. X., Meng F. Q., Guo S. Y., Zang G. H., and Jiang M. H., 1999, "Synthesis, structure and properties of a new nonlinear optical material: zinc cadmium tetrathiocyanate” Mater Res. Bull. Vol. 34, pp. 2003-2011.

3. Marcy H. O., Rosker M. J., Warren L., Cunningham P. H., Thomas C. A., Deloach L. A., Velsko S. P., Enners C. A., Liao J. H., and Kamatzidis M. G., 1995, "L-Histidine tetrafluoroborate: a solution-grown semiorganic crystal for nonlinear frequency conversion” Opt. Lett. Vol. 20, pp. 252-254.

4. Sastry P. U., Chitra R., Choudhury R. R., and Ramanadhan M., 2004,” Zinc (tris) thiourea sulphate (ZTS): A single crystal neutron diffraction study” Pramana J. of physics, 63, pp. 257-261.

5. Ushasree P. M., Jayavel R., Subramanian C., Ramasamy P., 1999, "Growth of zinc thiourea sulfate(ZTS) single crystals-a potential semiorganic NLO material”, J. Cryst. Growth, 197, pp. 216-220, 
6. Gupte S. J., and Desai C. F.,1999 " Vickers Hardness Anisotropy and Slip System in Zinc (Tris) Thiourea Sulphate Crystals”, Cryst. Res. Technol. 34, pp.1329-1332.

7. Ushasree P. M., Jayavel R., Ramasamy P., 1999, "Growth and Characterisation of phosphate mixed ZTS single crystals”, Mat. Science and Eng. B65, pp. 153-163.

8. Selvasekarapandian S., Vivekanandian K., Kolandaivel P., and Gundurao T. K., 1997, "Vibrational Studies of Bis(thiourea) Cadmium Chloride and Tris(thiourea) Zinc Sulphate Semiorganic Non-linear Optical Crystals”, Cryst. Res. Technol. 32, pp. 299-309.

9. Ushasree P.M., Jayavel R., Subramanian C. and Ramasamy P., 1999, "Growth of ZTS Single Crystal: A potential semiorganic NLO Material” J. Crystal Growth 197, pp. 216-223.

10. Silverstein R. M., Clayton Basseler G., and Morrill T. C., "Spectrometric Identificaton of Organic Compounds”, V-Edn. John Willey \& Sons, inc. New York 1998. 